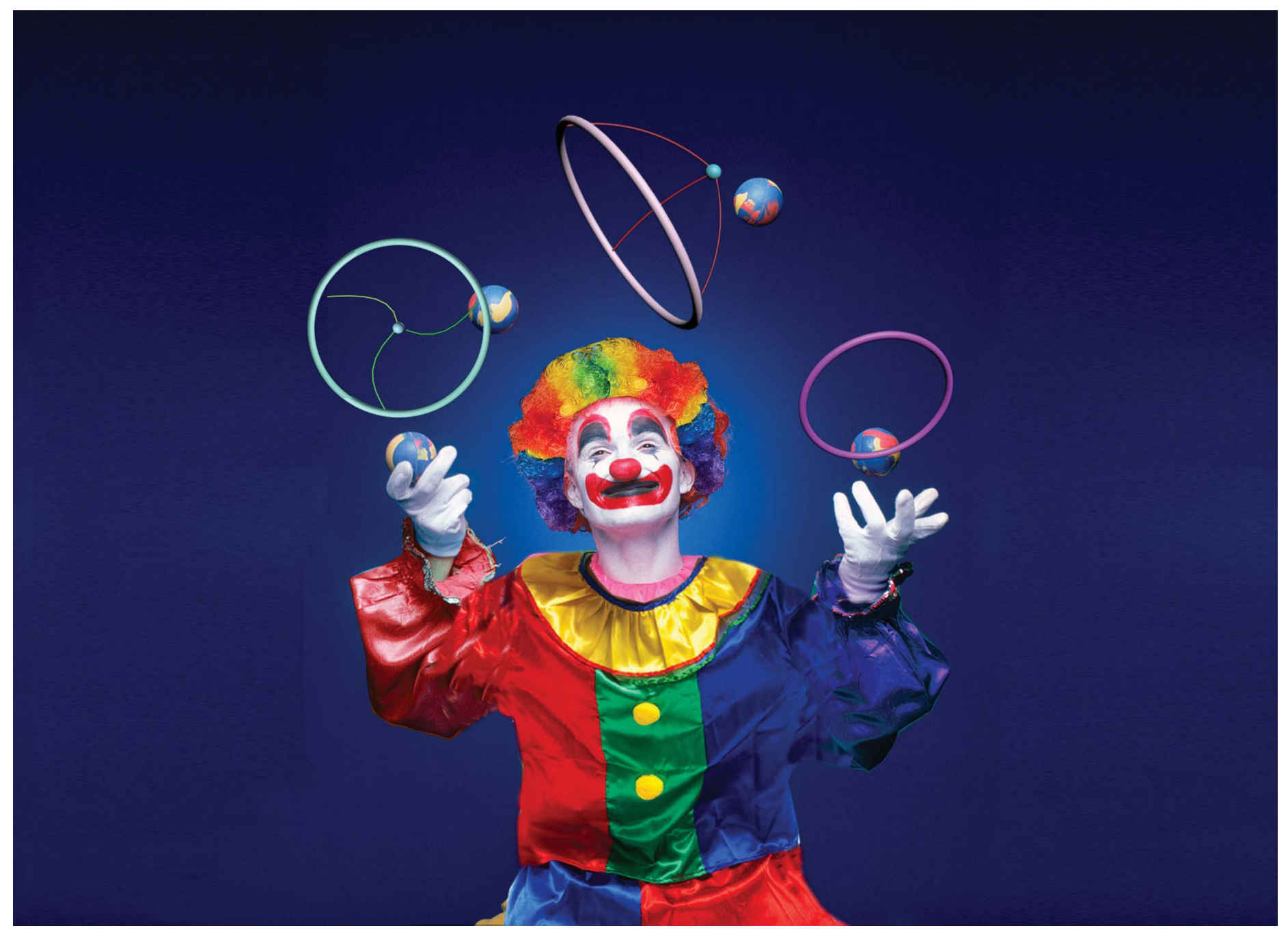

Showcasing research from Professor Hua Wei's laboratory, College of Chemistry and Chemical Engineering, Lanzhou University, Lanzhou, Gansu, P. R. China.

Synthesis of stimuli-responsive nanosized ring-like colloids and cyclic polymers via a dual-template approach

A dual-template approach was developed toward controlled synthesis of stimuli-responsive nanosized ring-like colloids and cyclic polymers using both a three-arm star-shaped polymer and $\beta$-cyclodextrin ( $\beta-C D$ ) as the templates. The dimension of the ring can be readily controlled by the molecular weight of the star polymers and the cross-linking degrees of the lipoic acid-functionalized $\beta-C D$. This study not only successfully builds a connection that bridges ring-like nanoparticles and cyclic polymers, but also opens a new avenue toward controlled synthesis of stimuli-responsive nanosized ring-like colloids and cyclic polymers for biomedical applications.

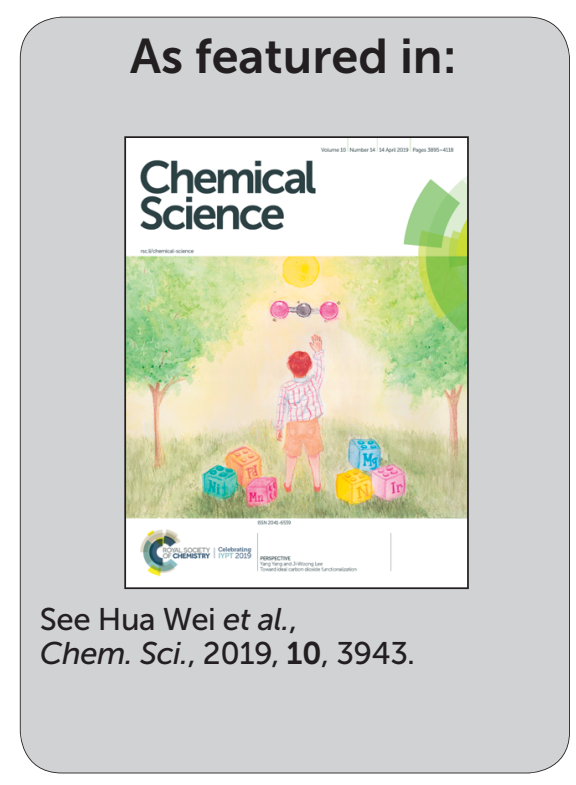


Check for updates

Cite this: Chem. Sci., 2019, 10, 3943

๑ All publication charges for this article have been paid for by the Royal Society of Chemistry
Received 26th January 2019

Accepted 4th March 2019

DOI: $10.1039 /$ c9sc00450e

rsc.li/chemical-science

\title{
Synthesis of stimuli-responsive nanosized ring-like colloids and cyclic polymers via a dual-template approach $\dagger$
}

\author{
Jinlei Peng, ${ }^{a}$ Cai Zuo, ${ }^{a}$ Qi Xiao, ${ }^{a}$ Kaicheng Deng, ${ }^{a}$ Chao Meng, ${ }^{a}$ Yuping Liu, ${ }^{a}$ \\ Miao Zhang, ${ }^{a}$ Liwei Ma, ${ }^{a}$ Suzie H. Pun ${ }^{b}$ and Hua Wei $\mathbb{D}$ *a
}

\begin{abstract}
Ring-like particles have received considerable attention due to their unique interior cavity and properties. However, the preparation of stimuli-responsive nanosized rings with internal size smaller than $100 \mathrm{~nm}$ remains unexplored likely due to the challenges encountered in their synthesis. The successful fulfillment of this target will not only significantly enrich the family of ring-like nanoparticles but also build a connection that bridges ring-like nanoparticles and cyclic polymers. For this purpose, we report in this study a controlled synthesis of stimuli-responsive ring-like colloids and cyclic polymers using both starshaped polymers and $\beta$-cyclodextrin $(\beta-C D)$ as the dual templates. The first template comprising starshaped polymers generated a ring-like structure and adoption of $\beta-C D$ as the second template further restricted the ring thickness to the height of a $\beta-C D$, leading to the generation of stimuli-responsive nanosized ring-like colloids with ring thickness less than $1 \mathrm{~nm}$, which shifted the ring-like structure to cyclic polymers with reversible cross-linked disulfide bridges. The reported "dual-template" approach is thus a valuable alternative to the current synthetic strategies toward stimuli-responsive ring-like colloids and cyclic polymers.
\end{abstract}

\section{Introduction}

In recent decades, great efforts have been made to explore the precise synthesis of nanoparticles with various controlled shapes $^{1-5}$ due to the critical effect of shape on the properties and performance of particles. ${ }^{6-8}$ Among the developed diverse shapes, the ring-like structure has attracted increasing attention because of its unique interior cavity and electromagnetic/ optical properties. ${ }^{9}$ Notable synthetic strategies leading to a ring-like structure include lithography, ${ }^{10}$ controlled wetting, ${ }^{11}$ hydrothermal processing, ${ }^{12}$ and protective etching, ${ }^{13}$ in which two critical parameters, i.e., ring size and thickness should be controlled. However, most of the synthesized ring-like structures showed a cavity of 100-1000 $\mathrm{nm}$ and a ring thickness in the range of nano to micro-scales. ${ }^{14-16}$ There are only a few reports, to the best of our knowledge, describing the synthesis of nanosized rings with internal size smaller than $100 \mathrm{~nm} \cdot{ }^{17,18} \mathrm{~A}$ recently notable example is Anderson's nanorings composed of

${ }^{a}$ State Key Laboratory of Applied Organic Chemistry, Key Laboratory of Nonferrous Metal Chemistry and Resources Utilization of Gansu Province, College of Chemistry and Chemical Engineering, Lanzhou University, Lanzhou, Gansu 730000, China. E-mail:weih@lzu.edu.cn

${ }^{b}$ Department of Bioengineering, Molecular Engineering and Sciences Institute, University of Washington, Seattle, Washington 98195, USA

$\dagger$ Electronic supplementary information (ESI) available. See DOI: $10.1039 / \mathrm{c} 9 \mathrm{sc} 00450 \mathrm{e}$ monodispersed cyclic porphyrin polymers with diameters of up to $21 \mathrm{~nm}^{18}$

For nanomedicine applications, drug carriers with approximately $100 \mathrm{~nm}$ in size have been reported to show 15 to 250 -fold higher uptake efficiency by the intestinal tissue relative to the micrometer-sized analogues. ${ }^{19}$ Therefore the successful fulfillment of this target will not only significantly enrich the family of ring-like nanoparticles but also build a connection that bridges ring-like nanoparticles and cyclic polymers, which will open a new avenue for the synthesis of cyclic polymers that is completely different from the classical strategies toward cyclic polymers based on ring-expansion polymerization (REP) and ring-closure reaction..$^{20-28}$ Moreover, stimuli-responsive rings capable of responding to various biological triggers, such as $\mathrm{pH}$, temperature, and reducing and oxidizing environments, have not been reported so far to our knowledge most likely due to the synthetic limitations. The simultaneous endowment of the resulting ringlike colloids with a nanosized dimension and stimuliresponsive properties will further promote the broad application of ring-like nanoparticles in nanomedicine. For this purpose, we report herein a controlled synthesis of stimuliresponsive nanosized ring-like colloids and cyclic polymers using both star-shaped polymers and $\beta$-cyclodextrin $(\beta-\mathrm{CD})$ as the dual templates.

Amphiphilic star polymers have a unique position in drug delivery systems due to their ability to assemble into 
unimolecular micelles with particle sizes less than $200 \mathrm{~nm} .{ }^{29,30}$ Star-shaped polymers with branched structures that were previously utilized as etching templates to prepare hollow nanoparticles ${ }^{31,32}$ were used to create a ring structure in this study. A three-arm star-shaped hydrophobic poly $(\varepsilon-$ caprolactone) (PCL) polymer was selected as an etching template for the following reasons: (i) the plane constructed by the three polymer termini of a three-arm star-shaped PCL was used to create a ring-like structure rather than a threedimensional sphere in this study given the axiom that the three points that are not on the same line lie in only one plane, (ii) star-shaped polymers have been reported to form unimolecular micelles with highly uniform structures and dimensions, ${ }^{29}$ and (iii) the size of the final ring can be tailored by the molecular weight (MW) of the polymers. Next, the thickness of a ring was always reported within the range of nano to micro-scales in contrast to the negligible thickness of typical cyclic polymers less than $1 \mathrm{~nm},{ }^{33,34}$ which further implies the synthetic challenge in shifting a ring-like structure to a cyclic polymer by controlling the ring thickness to a value smaller than $1 \mathrm{~nm}$. For this purpose, $\beta$-cyclodextrin ( $\beta$ $\mathrm{CD})$ was used as the second template in this study to generate a cyclic polymer with a ring thickness equal to the height of a $\beta$-CD. $\beta$-CD has been frequently used as a building block in biomaterials for its high water-solubility, biocompatibility, and ability to form inclusion complexes with hydrophobic entities such as certain small molecule drugs. ${ }^{3,36}$ In our design, $\beta$-CDs were modified with multiple oligoethylene glycol arms as well as lipoic acid (LA). Oligoethylene glycol modification improves the biocompatibility. ${ }^{37}$ LA allows for reversible dithiothreitol-mediated crosslinking of the cyclodextrins assembled around the star polymer template. The inclusion complexation ability of $\beta-C D^{38-41}$ is used to first modulate the formation of supramolecular star-shaped amphiphilic block copolymers and then to realize removal

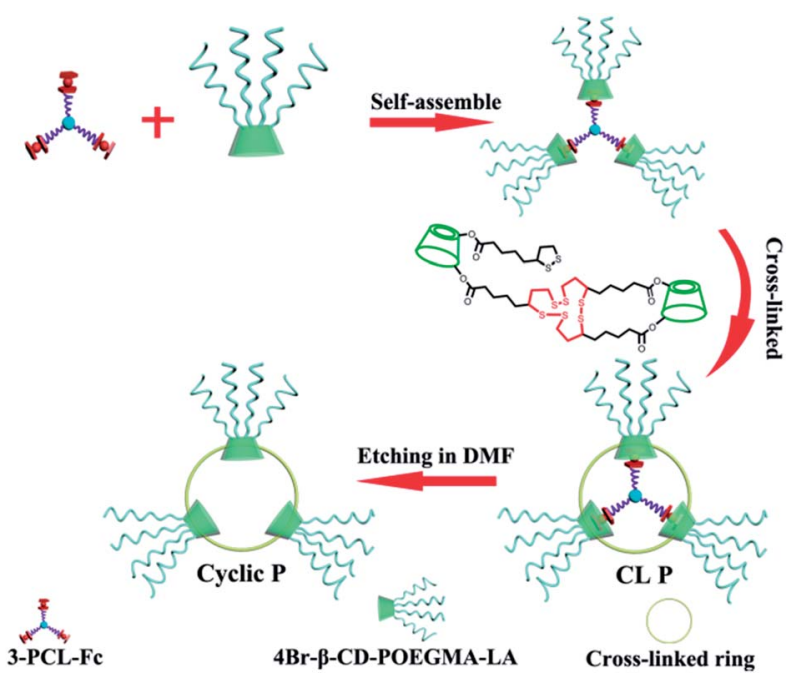

Scheme 1 Schematic illustration of a dual-template approach toward stimuli-responsive nanosized ring-like colloids and cyclic polymers. of the template by decomplexation, and finally to promote drug encapsulation (Scheme 1).

\section{Results and discussion}

\section{Synthesis of 3-PCL-Fc and 4Br- $\beta$-CD-POEGMA-LA}

The 4-arm poly(oligoethylene glycol) methacrylate (POEGMA) was synthesized by atom transfer radical polymerization (ATRP) using $\beta$-CD with four initiating sites as the multimacroinitiator (Scheme S1 $\dagger$ ). The statistically unmodified 17 hydroxyl groups in the primary and secondary faces of $4 \mathrm{Br}-\beta$-CD-POEGMA were further decorated with LA to afford $4 \mathrm{Br}-\beta$-CD-POEGMA-LA with two different degrees of substitution (DS) of LA. The DS of LA was calculated to be approximately 8 and 13, respectively. Meanwhile, the 3-arm PCL was prepared by ring-opening polymerization (ROP) using 2-(hydroxymethyl)propane-1,3-diol as an initiator, and the hydroxyl terminus of each arm was further decorated with ferrocene $(\mathrm{Fc})$ to produce 3-PCL-Fc (Scheme $\mathrm{S} 1 \dagger)$. The well-controlled synthesis of $4 \mathrm{Br}-\beta-\mathrm{CD}-\mathrm{POEGMA}-\mathrm{LA}$ and 3-PCL-Fc was confirmed by the unimodal and narrowdistributed SEC elution peaks for all the synthesized polymers (Fig. 1a and b).

The degree of polymerization (DP) of $4 \mathrm{Br}-\beta-\mathrm{CD}-\mathrm{POEGMA}-\mathrm{LA}$ and 3-PCL-Fc was calculated based on the previously assigned characteristic signals in the ${ }^{1} \mathrm{H}$ NMR spectra ${ }^{30}$ (Fig. S1-S8 in the $\mathrm{ESI} \dagger)$. The molecular parameters of all the synthesized polymers are summarized in Table $\mathrm{S} 1 . \dagger$

Preparation and characterisation of supramolecular micelles composed of one 3-PCL-Fc and three 4Br- $\beta$-CD-POEGMA-LA moieties

To demonstrate the precisely controlled ring size by the MW of the hydrophobic PCL core and the crosslinking density of LA, a series of three supramolecular star-shaped amphiphilic block copolymers with 12 arms and $\beta-\mathrm{CD} / \mathrm{Fc}$ noncovalent block junctions were facilely fabricated by quantitative host-guest complexations between $4 \mathrm{Br}-\beta$-CD-POEGMA-LA and 3-PCL-Fc with a molar feed ratio of 3 and 1 (Table 1). The host-guest recognition of $\beta-\mathrm{CD} / \mathrm{Fc}$ pairs was confirmed by the $2 \mathrm{D}$ NOESY spectrum, which shows the appearance of cross-peaks (highlighted in red) attributed to the dipolar interactions between the characteristic signals at 4.40 and $4.20 \mathrm{ppm}$ assigned to Fc and a)

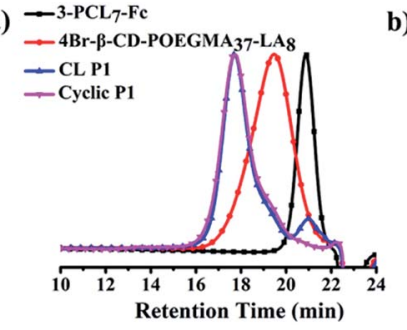

b)

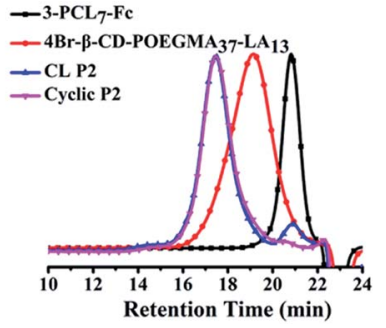

Fig. 1 SEC elution traces of (a) $3-\mathrm{PCL}_{7}-\mathrm{FC}, 4 \mathrm{Br}-\beta-\mathrm{CD}-\mathrm{POEGMA} \mathrm{A}_{37}$ $\mathrm{LA}_{8}, \mathrm{CL} \mathrm{P} 1$, cyclic P1 and (b) 3-PCL $-\mathrm{FC}, 4 \mathrm{Br}-\beta-\mathrm{CD}-\mathrm{POEGMA} \mathrm{A}_{37}-\mathrm{LA}_{13}$, CL P2, cyclic P2 using DMF as an eluent. 
Table 1 The hydrodynamic diameters of P1, P2 and P3 determined by DLS

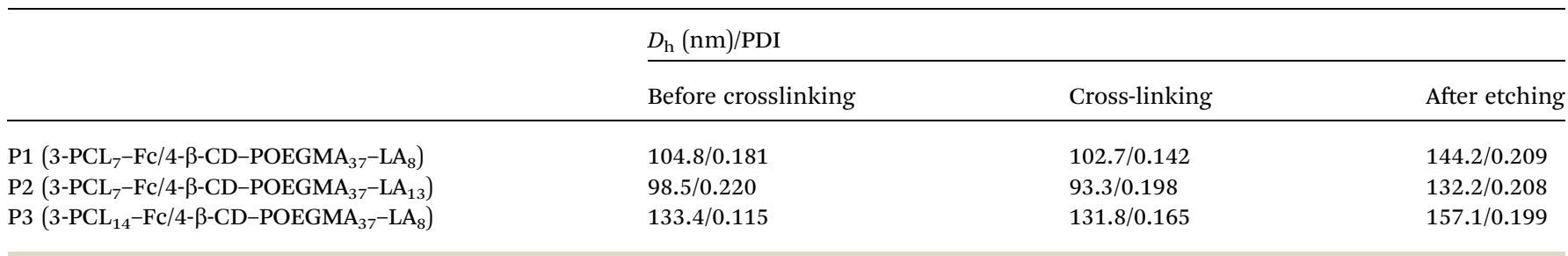

the resonance signals of $\beta-\mathrm{CD}$ in the range of $3.50-3.60 \mathrm{ppm}$ (Fig. S10†).

The formation of a supramolecular micelle containing only one 12-arm star-shaped amphiphilic block copolymer free of any aggregation of polymers in an aqueous phase is a crucial prerequisite for the subsequent production of uniform ring-like colloids. Dynamic light scattering (DLS) was used to determine the mean size of the self-assembled micelles (Table 1). The average size of micelles based on $3-\mathrm{PCL}_{7}-\mathrm{Fc} / 4-\beta-\mathrm{CD}-$ POEGMA $_{37}-\mathrm{LA}_{8}$ (P1) is $104.8 \mathrm{~nm}$ at a polymer concentration of $0.3 \mathrm{mg} \mathrm{mL}{ }^{-1}$. Given the hydrophobicity of PCL, the $\left\langle R_{\mathrm{g}}\right\rangle$ value of $3-\mathrm{PCL}_{7}-\mathrm{Fc}$ and $4-\beta$-CD-POEGMA ${ }_{37}-\mathrm{LA}_{8}$ determined by SECMALLS was used to estimate their respective dimensions instead of the hydrodynamic size determined by DLS in an aqueous phase. The $\left\langle R_{\mathrm{g}}\right\rangle$ value is $34.9 \mathrm{~nm}$ for $3-\mathrm{PCL}_{7}-\mathrm{Fc}$, and $26.5 \mathrm{~nm}$ for $4-\beta-\mathrm{CD}-\mathrm{POEGMA}{ }_{37}-\mathrm{LA}_{8}$. Therefore the contour length (diameter) of the micelles formed by $\mathrm{P} 1$ composed of 3$\mathrm{PCL}_{7}-\mathrm{Fc}$ and three $4-\beta-\mathrm{CD}-\mathrm{POEGMA}{ }_{37}-\mathrm{LA}_{8}$ was estimated to be $34.9+26.5 \times 2=87.9 \mathrm{~nm}$, which is close to the data $(104.8 \mathrm{~nm}$, Table 1 and Fig. S11 $\dagger$ ) determined by DLS. The formation of a micelle construct composed of only one star-shaped polymer with excellent stability was supported by the almost identical mean sizes and size distributions at various diluted concentrations of $0.01,0.05$, and $0.1 \mathrm{mg} \mathrm{mL}^{-1}$ (Fig. 2a) as well as an insignificant size change upon a shift of the solvents from water to DMF (Fig. S12a $\dagger$ ). The same capacities for $3-\mathrm{PCL}_{7}-\mathrm{Fc} / 4-\beta-\mathrm{CD}-$ a)

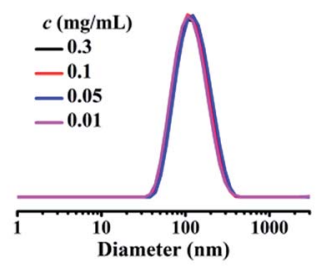

c)

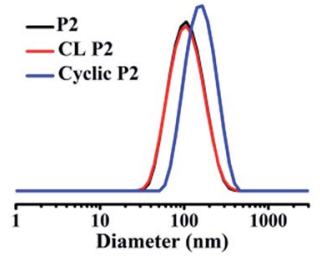

b)

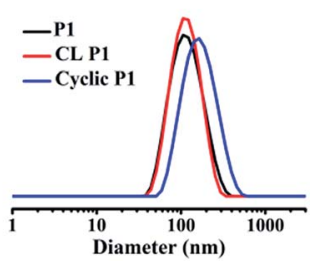

d)

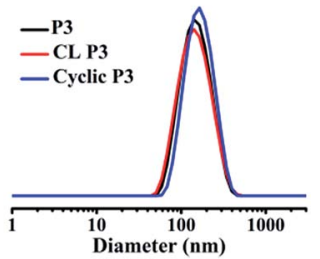

Fig. 2 DLS-determined intensity-average size distributions of (a) P1 at various concentrations ranging from 0.01 to $0.30 \mathrm{mg} \mathrm{mL}^{-1}$, (b) $\mathrm{P} 1, \mathrm{CL}$ P1, cyclic P1, (c) P2, CL P2, cyclic P2 and (d) P3, CL P3, cyclic P3 at $25^{\circ} \mathrm{C}$.
POEGMA $_{37}-\mathrm{LA}_{13}$ (P2) and $3-\mathrm{PCL}_{14}-\mathrm{Fc} / 4-\beta-\mathrm{CD}-\mathrm{POEGMA}{ }_{37}-\mathrm{LA}_{8}$ (P3) to form micelles with enhanced stability were confirmed by the similar results (Fig. S12b and $\mathrm{c} \dagger$ ). Note that the significantly large size (133.4 nm) of P3-based micelles among all of the three micelle constructs is reasonably attributed to the remarkably long hydrophobic PCL block of the P3-based micelles when compared to other micelles. The overall results imply the possibility of ultimately regulating the ring size of ring-like colloids by controlling the MW of the first template based on star-shaped PCL.

\section{Preparation and characterisation of crosslinked micelles}

Next, the LA functions decorated in the same and neighboring $\beta$-CDs underwent intermolecular crosslinking from the lipoic rings to the linear polydisulfides by ring-opening reactions in the presence of a catalytic amount of 1,4-dithio-D,L-threitol (DTT), ${ }^{42,43}$ leading to the formation of a micelle with a crosslinked band composed of three $\beta$-CD joints. It is important to note that the steric hindrance of the large hydrophilic stabilizing corona consisting of three hydrophilic 4-arm starshaped POEGMA moieties, in addition to the adopted concentration for stable micelle formation, guarantees the only occurrence of intramicelle cross-linking without any intermicelle associations, which is supported by the almost identical size before and after crosslinking (Fig. 2b-d). The freeze-dried crosslinked micelles were further subjected to SEC-MALLS analysis using $N, N$-dimethylformamide (DMF) as a solvent at $60{ }^{\circ} \mathrm{C}$ to investigate whether the crosslinking is sufficient because a locked band by efficient crosslinking is another prerequisite to ensure the structural integrity of the ring-like structure after the removal of the PCL core template. Interestingly, SEC-MALLS analysis of the crosslinked P1 (CL $\mathrm{P} 1$ ) could be used to monitor the etching process, which shows clearly bimodal SEC elution peaks centered at the elution times of approximately 18 and $21 \mathrm{~min}$, respectively (Fig. 1a, b, and S9c $\dagger$ ). Because the inclusion complex between $\beta$-CD and guest molecules was previously reported to dissociate in DMF at a relatively high temperature of $50{ }^{\circ} \mathrm{C},{ }^{44}$ the aforementioned main elution peak at a short retention time is reasonably attributed to the $\mathrm{CL} \mathrm{P} 1$ without the $3-\mathrm{PCL}_{7}-\mathrm{Fc}$ core, which matches well with the SEC elution peaks of the cyclic polymers. The other small elution peak with an identical retention time to that of $3-\mathrm{PCL}_{7}-\mathrm{Fc}$ is assigned to the dissociated 3$\mathrm{PCL}_{7}-\mathrm{Fc}$ moiety. More importantly, a clear shift of the elution peak of cyclic P1 toward shorter elution time and a calculated approximately 3-fold higher MW for cyclic P1 $\left(M_{\mathrm{n}}=96.8 \mathrm{kDa}\right.$, 
Table S1 $\dagger)$ relative to that of $4-\beta$-CD-POEGMA $37-\mathrm{LA}_{8}\left(M_{\mathrm{n}}=29.5\right.$ $\mathrm{kDa}$, Table $\mathrm{S} 1 \dagger)$ support successful formation of a ring containing three $4-\beta-\mathrm{CD}-\mathrm{POEGMA}{ }_{37}-\mathrm{LA}_{8}$ by sufficient crosslinking of a P1-based micelle. The slightly greater calculated ratio of 3.28 than the theoretical one of 3 is likely attributed to the molecular weight distribution of the synthesized polymers. The results also confirm the feasibility of etching the 3-PCL-Fc core from CL P1 using DMF and a relatively high temperature. The thickness of the crosslinked band was therefore confined to the height of $\beta$-CD at approximately $7.9 \AA^{45,46}$ Taken together, $\beta-C D$ in this step serves not only as a host for the construction of supramolecular star-shaped amphiphilic block copolymers, but also as the second template to restrict the thickness of the crosslinked band to a small value that is comparable to that of a cyclic polymer. Therefore the developed ring-like colloids are denoted as cyclic polymers in this study.

\section{Preparation and characterisation of cyclic polymers}

To finally generate a ring-like structure, the hydrophobic 3-arm PCL core was etched by an intensive dialysis in DMF at $50{ }^{\circ} \mathrm{C}$. The complete removal of the 3-PCL-Fc core from CL supramolecular star-shaped copolymers and successful production of cyclic polymers are confirmed by the complete loss of both a SEC elution peak attributed to the 3-PCL-Fc moiety and the characteristic resonance signals at $4.79,4.39$ and $4.18 \mathrm{ppm}$ (highlighted in red) assigned to the Fc segment in the SEC elution trace and ${ }^{1} \mathrm{H}$ NMR spectrum of the cyclic P1, respectively (Fig. 1 and 3). A panel of cyclic polymers with three different ring sizes was therefore successfully synthesized using an organic template with different MWs and cross-linking moieties with various crosslinking densities via a dual-template approach. A comparison of the average hydrodynamic sizes determined by DLS further revealed that a higher-MW 3-PCL-Fc template and a lower crosslinking density of LA moieties result in a larger ring size of the generated cyclic polymers (Table 1). For example, cyclic P1 and P2 share the same etching template but different crosslinking LA units. Therefore the higher crosslinking density due to a larger amount of LA units

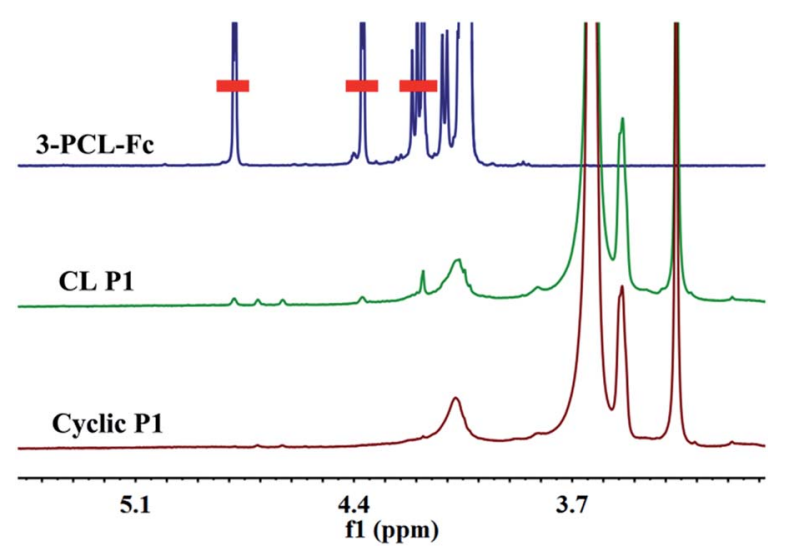

Fig. $3{ }^{1} \mathrm{H}$ NMR spectra of 3-PCL-Fc, CL P1, and cyclic P1 in $\mathrm{CDCl}_{3}$. accounts for a more compact ring structure with a smaller ring size for cyclic P2. All the three cyclic polymers show significantly increased hydrodynamic size compared to the CL formulations, which is likely attributed to the removal of the hydrophobic PCL core for the swelling of the hydrophilic locked band containing three 4 - $\beta$-CD-POEGMA ${ }_{37}$-LA.

TEM measurements were performed to visualize the morphological changes of the nanoobjects acquired at each preparation stage (Fig. 4 and S13-S15†). Taking the P1-based system for example, the micelles self-assembled by P1 are well-dispersed with regularly round shape and quite uniform diameter at approximately $30 \pm 3.7 \mathrm{~nm}$ under TEM observation. The CL P1 micelles show almost identical morphology and size to $\mathrm{P} 1$ micelles. Theoretical calculation regarding the range of the contour length of the etching template comprising threearm star-shaped PCL was performed (ESI $\dagger$ ) to verify the dimensions observed by TEM. The TEM observation supports the mere occurrence of intramicellar cross-linking as well. After etching of the 3-PCL-Fc core, the resulting cyclic P1 shows clearly hollow structures with highly uniform size and morphology (Fig. 4c), which is quite different from the images of CL P1. The swelling of the cyclic P1 after removal of the hydrophobic core is obviously reflected by an increase of the particulate size to approximately $40 \pm 6.1 \mathrm{~nm}$. AFM measurements were further employed to characterize the molecular topology of cyclic polymers. The clear observation of uniform rings (Fig. 4d, S13, S16, and S17†) convincingly supports the successful generation of cyclic polymers after core etching. The average size of cyclic P1 is about $38 \pm 4.3 \mathrm{~nm}$, which matches well with the results obtained by TEM analysis. The average height of CL P1 is about $0.5 \mathrm{~nm}$ on a Si substrate (Fig. S16 $\dagger$ ), and decreases to about $0.3 \mathrm{~nm}$ after core etching (Fig. S17†). Note that the captured blurred AFM height images are most likely attributed to the low height of the cyclic polymer, which agrees well with the previously reported height of the typical cyclic polymers. ${ }^{47}$ First of all, the differences between the ring size
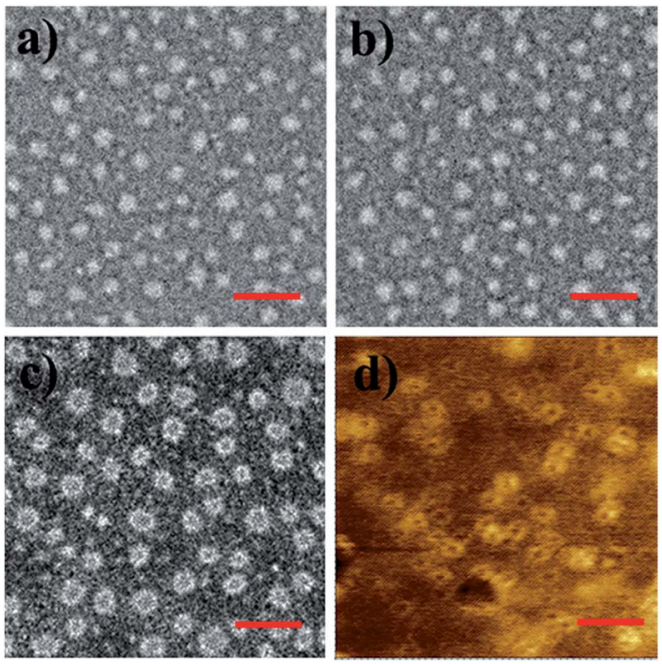

Fig. 4 TEM images of (a) P1, (b) CL P1, and (c) cyclic P1. AFM phase image of (d) cyclic P1 on a silicon wafer (scale bar: $100 \mathrm{~nm}$ ). 
a)
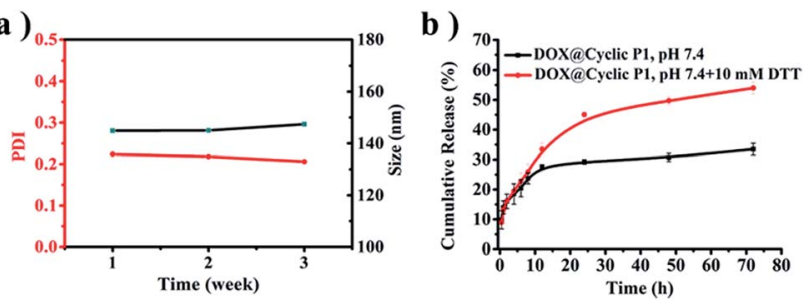

Fig. 5 (a) Intensity-average sizes and PDIs from DLS of cyclic P1 at an incubation period of three weeks at $37^{\circ} \mathrm{C}$. (b) In vitro drug release profiles of DOX@cyclic P1.

measured by DLS and microscopy observations are attributed to the different status, i.e., hydration and dried conditions adopted for the two analytical techniques, respectively. The addition of a hydrated shell accounts for the larger size of the nanoparticles revealed by DLS relative to that observed by TEM. Moreover, there are three different calculation modes employed for DLS analysis, the intensity, number and volume-average size distributions, respectively. A comparison of the three different size distributions (Fig. S11†) acquired for P1 at a polymer concentration of $0.30 \mathrm{mg} \mathrm{mL} \mathrm{mL}^{-1}$ clearly shows a population centered at the smallest size of approximately $50 \mathrm{~nm}$ with the narrowest size distribution for the number-average size plot. Taken together, the relatively large differences between the sizes determined by TEM and by DLS were ascribed to the different states of the samples used for the two measurements as well as the different calculation modes used for the DLS analysis.

\section{In vitro drug loading and drug release study}

The salt stability of cyclic P1 in PBS (pH 7.4, $150 \mathrm{mM}$ ) is supported by the negligible variations of both average size and polydispersity index (PDI) during an incubation period of three weeks (Fig. 5a). Due to the presence of crosslinked disulfide bridges that can respond to the intracellular reducing environment of tumor cells ${ }^{48}$ in the resulting cyclic polymers, the potential biomedical applications of cyclic P1 were further evaluated by an in vitro drug release study using doxorubicin (DOX) as a model drug. The drug loading content (DLC) and entrapment efficiency (EE) of DOX-loaded cyclic P1 (DOX@cyclic P1) prepared by a classical dialysis method were $4.3 \%$ and $41.0 \%$, respectively. The drug release profiles were evaluated at the physiological $\mathrm{pH}$ of 7.4 and the reducing environments of tumor cells (pH 7.4, $10 \mathrm{mM}$ DTT), respectively (Fig. 5b). Incubation at $\mathrm{pH} 7.4$ led to a $33.5 \%$ DOX release in $72 \mathrm{~h}$. However, a shift of the release media to pH 7.4 with $10 \mathrm{mM}$ DTT significantly promoted the cumulative drug release to $54.0 \%$ in the same duration, which is attributed to the reduction-triggered dissociation of cyclic polymers containing three covalently bonded cyclodextrin units toward an accelerated drug release.

\section{Conclusions}

In summary, we have successfully developed a dual-template approach toward the controlled synthesis of stimuliresponsive nanosized ring-like colloids and cyclic polymers using both a three-arm star-shaped polymer and $\beta$-cyclodextrin $(\beta-\mathrm{CD})$ as the templates. We further demonstrated that the dimension of the ring can be readily controlled by the MW of the star polymers as well as the cross-linking degrees of LA functions. We believe that this study not only successfully builds a connection that bridges ring-like nanoparticles and cyclic polymers, but also opens a new avenue toward controlled synthesis of stimuli-responsive nanosized ring-like colloids and cyclic polymers for biomedical applications.

\section{Conflicts of interest}

There are no conflicts to declare.

\section{Acknowledgements}

The authors acknowledge the financial support from the National Natural Science Foundation of China (51473072 and 21504035), the Thousand Young Talent Program, and the Open Research Fund of State Key Laboratory of Polymer Physics and Chemistry, Changchun Institute of Applied Chemistry, Chinese Academy of Sciences.

\section{Notes and references}

1 G. H. Gweon, T. Sasagawa, S. Y. Zhou, J. Graf, H. Takagi, D. H. Lee and A. Lanzara, Nature, 2004, 430, 187-190.

2 A. Kuijk, A. van Blaaderen and A. Imhof, J. Am. Chem. Soc., 2011, 133, 2346-2349.

3 J. Liang, X. Y. Yu, H. Zhou, H. B. Wu, S. Ding and X. W. Lou, Angew. Chem., Int. Ed., 2014, 53, 12803-12807.

4 J. W. Kim, R. J. Larsen and D. A. Weitz, Adv. Mater., 2007, 19, 2005-2009.

5 Y. Mato, K. Honda, K. Tajima, T. Yamamoto, T. Isono and T. Satoh, Chem. Sci., 2019, 10, 440-446.

6 M. Zamani, M. P. Prabhakaran and S. Ramakrishna, Int. J. Nanomed., 2013, 8, 2997-3017.

7 P. Datskos and J. Sharma, Angew. Chem., Int. Ed., 2014, 53, 451-454.

8 E. Paineau, M. E. Krapf, M. S. Amara, N. V. Matskova, I. Dozov, S. Rouziere, A. Thill, P. Launois and P. Davidson, Nat. Commun., 2016, 7, 10271.

9 A. Lorke, R. J. Luyken, A. O. Govorov and J. P. Kotthaus, Phys. Rev. Lett., 2000, 84, 2223-2226.

10 J. M. McLellan, M. Geissler and Y. Xia, J. Am. Chem. Soc., 2004, 126, 10830-10831.

11 H. Xu and W. A. Goedel, Angew. Chem., Int. Ed., 2003, 42, 4696-4700.

12 X. Hu, J. C. Yu, J. Gong, Q. Li and G. Li, Adv. Mater., 2007, 19, 2324-2329.

13 F. Yan and W. A. Goedel, Angew. Chem., Int. Ed., 2005, 44, 2084-2088.

14 S. Jung, W. Cho, H. J. Lee and M. Oh, Angew. Chem., Int. Ed., 2009, 48, 1459-1462.

15 Y. Wu, Z. Luo, B. Liu and Z. Yang, Angew. Chem., Int. Ed., 2017, 56, 9807-9811. 
16 S. Mei, H. Qi, T. Zhou and C. Y. Li, Angew. Chem., Int. Ed., 2017, 56, 13645-13649.

17 W. J. Cho, A. Jeremic and B. P. Jena, J. Am. Chem. Soc., 2005, 127, 10156-10157.

18 D. V. Kondratuk, L. A. Perdigao, A. M. S. Esmail, J. N. O'Shea, P. H. Beton and H. L. Anderson, Nat. Chem., 2015, 7, 317322.

19 M. P. Desai, V. Labhasetwar, G. L. Amidon and R. J. Levy, Pharm. Res., 1996, 13, 1838-1845.

20 C. W. Bielawski, D. Benitezand and R. H. Grubbs, J. Am. Chem. Soc., 2003, 125, 8424-8425.

21 M. Schappacher and A. Deffieux, Science, 2008, 319, 15121515.

22 Y. Xia, A. J. Boydston and R. H. Grubbs, Angew. Chem., Int. Ed., 2011, 50, 5882-5885.

23 K. Zhang, M. A. Lackey, Y. Wu and G. N. Tew, J. Am. Chem. Soc., 2011, 133, 6906-6909.

24 B. A. Laurent and S. M. Grayson, Chem. Soc. Rev., 2009, 38, 2202-2213.

25 B. A. Laurent and S. M. Grayson, J. Am. Chem. Soc., 2006, 128, 4238-4239.

26 B. J. Lidster, S. Hirata, S. Matsuda, T. Yamamoto, V. Komanduri, D. R. Kumar, Y. Tezuka, M. Vacha and M. L. Turner, Chem. Sci., 2018, 9, 2934-2941.

27 Z. Liu, Y. Huang, X. Zhang, X. Tu, M. Wang, L. Ma, B. Wang, J. He, P. Ni and H. Wei, Macromolecules, 2018, 51, 7672-7679.

28 X. Y. Tu, M. Z. Liu and H. Wei, J. Polym. Sci., Part A: Polym. Chem., 2016, 54, 1447-1458.

29 X. Jin, P. Sun, G. Tong and X. Zhu, Biomaterials, 2018, 178, 738-750.

30 C. Zuo, J. Peng, Y. Cong, X. Dai, X. Zhang, S. Zhao, X. Zhang, L. Ma, B. Wang and H. Wei, J. Colloid Interface Sci., 2018, 514, 122-131.

31 X. Pang, L. Zhao, W. Han, X. Xin and Z. Lin, Nat. Nanotechnol., 2013, 8, 426-431.

32 C. Feng, X. Pang, Y. He, B. Li and Z. Lin, Chem. Mater., 2014, 26, 6058-6067.
33 E. J. Shin, H. A. Brown, S. Gonzalez, W. Jeong, J. L. Hedrick and R. M. Waymouth, Angew. Chem., Int. Ed., 2011, 50, 63886391.

34 H. Wei, C. E. Wang, N. Tan, A. J. Boydston and S. H. Pun, ACS Macro Lett., 2015, 4, 938-941.

35 J. Jing, A. Szarpak-Jankowska, R. Guillot, I. Pignot-Paintrand, C. Picart and R. Auzély-Velty, Chem. Mater., 2013, 25, 38673873.

36 X. Song, Y. Wen, J. L. Zhu, F. Zhao, Z. X. Zhang and J. Li, Biomacromolecules, 2016, 17, 3957-3963.

37 I. Ozer, A. Tomak, H. M. Zareie, Y. Baran and V. Bulmus, Biomacromolecules, 2017, 18, 2699-2710.

38 P. Du, J. Liu, G. Chen and M. Jiang, Langmuir, 2011, 27, 96029608.

39 L. Peng, A. Feng, M. Huo and J. Yuan, Chem. Commun., 2014, 50, 13005-13014.

40 B. V. K. J. Schmidt, D. Kugele, J. von Irmer, J. Steinkoenig, H. Mutlu, C. Rüttiger, C. J. Hawker, M. Gallei and C. Barner-Kowollik, Macromolecules, 2017, 50, 2375-2386.

41 B. V. K. J. Schmidt and C. Barner-Kowollik, Angew. Chem., Int. Ed., 2017, 56, 8350-8369.

42 A. Sadownik, J. Stefely and S. L. Regen, J. Am. Chem. Soc., 1986, 108, 7789-7791.

43 E. K. Bang, M. Lista, G. Sforazzini, N. Sakai and S. Matile, Chem. Sci., 2012, 3, 1752-1763.

44 J. Wang and M. Jiang, J. Am. Chem. Soc., 2006, 128, 37033708.

45 L. Wang, L. L. Li, Y. S. Fan and H. Wang, Adv. Mater., 2013, 25, 3888-3898.

46 C. Zuo, X. Dai, S. Zhao, X. Liu, S. Ding, L. Ma, M. Liu and H. Wei, ACS Macro Lett., 2016, 5, 873-878.

47 A. J. Boydston, T. W. Holcombe, D. A. Unruh, J. M. J. Fréchet and R. H. Grubbs, J. Am. Chem. Soc., 2009, 131, 5388-5389.

48 M. H. Lee, Z. Yang, C. W. Lim, Y. H. Lee, S. Dongbang, C. Kang and J. S. Kim, Chem. Rev., 2013, 113, 5071-5109. 